\section{WHOLE GENOME EXPRESSION IN VERY LOW BIRTHWEIGHT (VLBW) INFANTS WITH AND WITHOUT RETINOPATHY OF PREMATURITY (ROP) - PRELIMINARY RESULTS}

J.J. Pietrzyk ${ }^{1}$, P. Kwinta ${ }^{1}$, M. Bik-Multanowski², A. Madetko-Talowska ${ }^{2}$, T. Tomasik ${ }^{1}$, M. Jagła ${ }^{1}$, Z. Mitkowska ${ }^{1}$, E.J. Wollen ${ }^{3}$, O.D. Saugstad ${ }^{3}$

${ }^{1}$ Department of Pediatrics, ${ }^{2}$ Department of Medical Genetics, Jagiellonian University Medical College, Cracow, Poland, ${ }^{3}$ Department of Pediatric

Research, University of Oslo, Rikshospitalet, Oslo, Norway

Introduction: At present ROP is the most common cause of blindness in children. Owing to the microarray technique, expression of all potential human and animal genes may be reliably evaluated.

Aim: To compare whole genome expression in the first month of life in the groups of infants with and without ROP.

Methods: 33 VLBW newborns (mean birthweight 1047g (SD:272), mean gestational age 27.9 weeks (SD: 2.7)) were prospectively evaluated. Blood samples were drawn from all the study participants on the $5^{\text {th }}, 14^{\text {th }}$ and $28^{\text {th }}$ days of life (DOL). AMBION RiboPure Blood kits were used to extract mRNA. The mRNA samples were evaluated for gene expression with the use of GeneChip $®$ Human Gene 1.0ST microarrays. The infants were divided into 2 groups: A) no ROP or ROP not requiring treatment $(n=22)$ and 2) ROP requiring laser therapy $(n=11)$.

Results: 669 genes were significantly differentially expressed between the groups during the first month of life ( 325 genes on the $5^{\text {th }}$ DOL, 269 on the $14^{\text {th }} \mathrm{DOL}$ and 260 on $\left.28^{\text {th }} \mathrm{DOL}\right) .16$ genes were consistently overexpressed and 15 genes were consistently underexpressed in all three measurements. Preliminary gene ontology analyses show that the genes expressed differently are mainly these involved in cell growth and differentiation.

Conclusion: Significant difference in 669 genes expression in the groups of infants with ROP were found. The impact of the over- and underexpressed genes needs further analysis.

Study supported by Financial Mechanism of European Economic Area (PL0226) and Polish Ministry of Science (E023/P01/2008/02/85)

\section{NEUROLOGICAL DAMAGE PREDICTION BY FETAL DOPPLER PARAMETERS IN INTRAUTERINE GROWTH RESTRICTED PRETERM INFANTS}

V. Tenorio ${ }^{1,2,3}$, R. Cruz-Martinez ${ }^{1,2,3}$, F. Figueras ${ }^{1,2,3}$, F. Crispi ${ }^{1,2,3}$, A. Arranz ${ }^{1,2,3}$, D.V. Valsky ${ }^{1,2,3}$, E. Hernandez-Andrade ${ }^{1,2,3}$, E. Gratacos ${ }^{1,2,3}$

${ }^{1}$ Maternal-Fetal and Neonatology Departments, Institut Clinic of Gynecology, Obstetrics and Neonatology, Hospital Clinic-Universidad de Barcelona, ${ }^{2}$ Fetal and Perinatal Medicine Research Group, Institut d'Investigacions Biomèdiques August Pi i Sunyer (IDIBAPS), ${ }^{3}$ Centro de Investigación Biomédica en Red de Enfermedades Raras (CIBER-ER), Instituto de Salud Carlos III, Barcelona, Spain

Background and aims: Intrauterine growth restriction (IUGR) increases the risk for adverse neurodevelopment. While prenatal prediction of mortality is now greatly improved, prediction of neurodevelopmental morbidity remains limited. We explored the value of fetal cardiovascular Doppler parameters to predict neonatal brain damage and abnormal neurobehavior in IUGR.

Methods: Doppler parameters (DV pulsatility index (PI), MPI, and Aol PI) were performed in a cohort of IUGR preterm (< 35 weeks) fetuses with abnormal umbilical artery Doppler. Neonates underwent several cranial ultrasound scans and the NeonatalBehavioral-Assessment-Scale (NBAS) at 40 weeks of corrected age. Isolated and combined value of cardiovascular parameters to predict interventricular hemorrhage (IVH), periventricular leukomalacia (PVL), basal ganglia damage (BGD), and abnormal NBAS were evaluated by multiple logistic regression and decision tree analysis. Gestational age (GA), middle cerebral artery, and umbilical artery were included as covariates.

Results: Of 106 IUGR fetuses, 90 survivors were studied. Mean GA at birth was 30.9 weeks. Retrograde flow in the DV and the Aol were significantly associated with brain injury with adjusted odds ratios (OR) of 8.6 and 4.1 respectively. Decision tree analysis combining these parameters discriminated high (67\%), moderate (52\%), and low risk (12\%) for any brain lesion at 40 weeks. Retrograde Aol PI identified a high risk of abnormal NBAS in habituation (OR 14.4) and attention capacity (OR 12.6). 